building, and absorption, (3) policy and product development, (4) health benefits, and (5) broader economic benefits. DISCUSSION/SIGNIFICANCE OF IMPACT: This study will aid in characterizing the returns resulting from this research funding and identify its strengths and weaknesses. This study will inform our understanding of the diversity and breadth of outcomes resulting from Georgia CTSA-supported research, and the value pilot projects provide to clinical and translational science and the broader community.

\section{Assessing research impact: It takes a team}

Ashley Dunn and Michelle B. Bass

Stanford University School of Medicine

OBJECTIVES/SPECIFIC AIMS: Dissemination of research findings through the published literature is a complex but critical part of the scholarly communication process. Additionally, this time point on the translational spectrum is a key objective of the National Clinical Association for Advancing Translational Sciences (NCATS). Tracking the dissemination of research outputs can be difficult to identify and evaluate. The purpose of this case study was 2-fold: (I) identify tools and resources available freely to the public and through university subscriptions used to assess research output; and (2) compare the effectiveness of these tools oat tracking output at different levels of granularity. METHODS/STUDY POPULATION: The authors, Spectrum staff (D.A.) and School of Medicine librarian (M.B.), attended webinars hosted by other Academic Medical Center libraries conducting work on impact tracking and learned from vendor product managers about available tools and resources during on-site campus visits. Publications from Stanford's Clinical and Translational Science Award (CTSA) were used to track the diffusion of research outputs (e.g., number of citations, document types, research areas, relative citation ratio, CTSAs collaboration) via library subscription services (e.g., Web of Science and Scopus) and freely available tools (e.g., iCite and PubMed). RESULTS/ANTICIPATED RESULTS: The authors found certain tools were more inclusive in retrieving grant funded research outputs. For example, in the case of ULI grant (UL ITR00 1085, UL I TR000093, UL IRR025744), on a grantlevel output, there were discrepancies in the number of publications retrieved: (I) PubMed found 644 outputs; (2) Web of Science found 497 outputs; and (3) Scopus found 190 outputs. After de-duplication, the search across Web of Science (WoS), Scopus, and PubMed yielded 899 publications. In total, 389 outputs were unique to PubMed; I 65 were unique to WoS; and 90 were unique to Scopus. Future analysis will be conducted to identify the source of unique outputs from each database (e.g., conference proceeding, specific journals). Additional analysis based on other units of research outputs (e.g., author-level outputs and article-level outputs) are expected to yield similar discrepancies. DISCUSSION/SIGNIFICANCE OF IMPACT: Citation analysis is a valuable method of assessing research output and, to a larger extent, research impact in a given field. It can help investigators illustrate qualifications for undertaking new projects, highlight collaborations across schools and departments, justify a grant renewal, and/or highlight accomplishments for promotion. However, systematic and comprehensive evaluations are needed in tandem with citation analysis/bibliometric analysis to assess the translation and uptake of research outputs and activities that result in research impact. Furthermore, both investigators and staff need adequate time and training to process research outputs/activities and to effectively organize them in easily understood visualizations.

Attitudes and preferences for return of results from next-generation sequencing

Matthew Neu, Jaimie Richards and Sara J. Knight

University of Alabama at Birmingham

OBJECTIVES/SPECIFIC AIMS: Objectives: Decreasing costs and increasing evidence for clinical utility have contributed to whole genome sequencing (WGS) becoming a clinical reality. While previous studies have surveyed the attitudes of patients and community members towards specific gene tests, an emerging literature has begun to describe the preferences of diverse recipients for WGS results. In this study, we sought to identify and synthesize the quantitative evidence on preferences for results from WGS using a systematic review of the literature. METHODS/STUDY POPULATION: We conducted a search of articles on PubMed including subject index terms WGS, whole exome sequencing, genome sequencing, secondary findings, incidental findings, attitudes, preferences, choices, utilities, stated-preferences, discrete choice experiment, and willingness-to-pay. We conducted II formal searches to refine the strategy and conducted a final search in December 2017. Duplicates were eliminated and a title and abstract review was conducted to select articles meeting inclusion criteria. RESULTS/ANTICIPATED RESULTS: Our search strategy identified 79 publications meeting initial search criteria with 30 manuscripts meeting inclusion criteria. Of these, most studies were conducted with patientparticipants enrolled in existing sequencing studies, while few engaged members of the general public. Of the studies conducted on patients, most were on the medical setting of cancer and related syndromes. The earliest publication date of a manuscript meeting our inclusion criteria was in 2012, yet the majority were published in 2015 or later. DISCUSSION/SIGNIFICANCE OF IMPACT: Between 2012 and 2015, we saw an increasing focus in the medical literature on understanding public and patient preferences for return of results from WGS and WES. Both public and patient populations participating in surveys expressed preferences for receiving results from next-generation sequencing, even if the results are secondary or incidental findings unrelated to the primary indication for sequencing. A primary factor related to patient interest in incidental or secondary findings is the extent to which these results can inform medical intervention. Few studies surveyed representative populationbased samples, and this may be an area for future investigation.

Balancing patient-centeredness and patient safety in the hospitals: The case of pain care and patient satisfaction Olena Mazurenko', Basia Andraka-Christou ${ }^{2}$, Matthew Bair ${ }^{3}$, Areeba $\mathrm{Kara}^{3}$ and Christopher A. Harle ${ }^{3}$

${ }^{1}$ Indiana University School of Medicine; ${ }^{2}$ Florida University; ${ }^{3}$ Indiana University

OBJECTIVES/SPECIFIC AIMS: This study seeks to understand the relationship between opioid prescribing and patient satisfaction among non-surgical, hospitalized patients. As part of this study, we qualitatively examined challenges in delivering safe and patient-centered care through voices of physicians', and nurses.' METHODS/STUDY POPULATION: We collected data through in-person interviews using semi-structured guides tailored to the informant roles. Study participants came from I healthcare system located in a mid-Western state. Each interview lasted 30-45 minutes, was audio-recorded with consent, and transcribed for analysis. Two researchers each coded 17 transcripts for discussions around patient-centeredness (including patient satisfaction, patient experiences), and patient safety for hospitalized patients experiencing pain. Analysis followed a general inductive approach, where researchers identified themes related to the research questions using an open coding technique. They discussed and reached consensus on all codes, and extracted several preliminary themes. The analysis was supported by NVivo software. RESULTS/ANTICIPATED RESULTS: The following themes emerged: (I) complex decision-making process to prescribe opioids for hospitalized patients; (2) the role of objective findings in prescribing decisions; (3) bargaining process in prescribing opioids; (4) balancing patient-centeredness and patient safety for selected populations; (5) opioids are the predominant medications for pain care. DISCUSSION/SIGNIFICANCE OF IMPACT: Clinicians' decision to prescribe opioids for nonsurgical hospitalized patients is based on multiple factors, including patient's condition, patient's preference for pain medications, or standard hospital's pain care regimen. Interventions that improve clinicians' ability to prescribe opioids may be needed to improve delivery of patient-centered and safe pain care.

Cost effectiveness analysis of operative Versus antibiotic management for uncomplicated appendicitis Eric Stulberg', Alexander Zheutlin, Raymond Strobel, Katherine $\mathrm{He}^{2}$ and Adelyn Beil ${ }^{2}$

${ }^{1}$ Northwestern University; ${ }^{2}$ University of Michigan School of Medicine

OBJECTIVES/SPECIFIC AIMS: (I) Evaluate the relative incremental costeffectiveness [cost per quality-adjusted life year (QALY) gained] of antibiotics, laparotomy, and laparascopy for the initial treatment of uncomplicated appendicitis. (2) Detect if the relative incremental cost-effectiveness of each treatment differs by age, namely in pediatric patients, adult patients, and geriatric patients. (3) Use deterministic and probabilistic sensitivity analyses to assess the robustness of our findings when varying multiple model parameters. METHODS/STUDY POPULATION: Study Population and Analytic Approach: The population under analysis is a simulated population of those aged I-90 diagnosed with uncomplicated appendicitis with computed tomography (CT) in the emergency department. Pregnant women and those younger than I year old were excluded from our analysis. We simulated our population through a Markov state-transition simulation model. Using this model, we estimated the lifelong costs and effects on QALYs from the use of antibiotics, laparoscopy, and 